\title{
Influencia de la Tipología del Ligante en las Propiedades Mecánicas de una Mezcla Asfáltica Densa
}

\author{
Oscar J. Reyes-Ortiz ${ }^{1}$, Gonzalo A. Valdés ${ }^{2^{*}}$ y Mario Salazar ${ }^{3}$ \\ (1) Universidad Militar de Nueva Granada, Departamento de Ingeniería Civil - Grupo Geotecnia, \\ Carera 11 № 101-80, Bogotá-Colombia \\ (2) Universidad de La Frontera, Departamento de Ingeniería de Obras Civiles, Francisco Salazar \\ No 01145, Temuco-Chile (e-mail: gvaldes@ufro.cl) \\ (3) Univ. Michoacana, Departamento de Ingeniería Civil, Av. Francisco Mujica s/n, Morelia-México
}

* autor a quien debe ser dirigida la correspondencia

\begin{abstract}
Resumen
En el presente artículo se presentan los resultados del estudio destinado a analizar la influencia del tipo de ligante en las propiedades mecánicas de una mezcla asfáltica densa. Los ligantes asfálticos utilizados en el estudio fueron dos ligantes de penetración, CA 60/80 y CA 80/100, y estirenobutadieno-estireno (SBS), un ligante modificado con polímeros. Las propiedades mecánicas analizadas para cada ligante evaluado en la mezcla fueron: cohesión, módulo resiliente, resistencia a la tracción indirecta y deformaciones plásticas. Los resultados permiten concluir la importancia de la utilización del ligante modificado en este tipo de mezclas, puesto que presentan un mejor comportamiento frente a las propiedades mecánicas evaluadas. Se establece también que la energía de compactación y la temperatura de ensayo tienen una mayor influencia en las propiedades mecánicas de las mezclas fabricadas con ligantes de penetración respecto a las fabricadas con el ligante modificado.
\end{abstract}

Palabras clave: mezcla asfáltica, módulo resiliente, deformaciones pláticas, propiedades mecánicas

\section{Influence of Asphalt Type on the Mechanical Properties of a Dense Asphalt Mixture}

\begin{abstract}
This paper presents the analyses of the influence of different asphalt types on the mechanical properties of a dense asphalt mixture. Two penetration binders, CA 60/80 and CA 80/100, and styrene-butadiene-styrene (SBS) a modified polymer binder were used. Mechanical properties as cohesion, resilient modulus, indirect tensile resistance and rutting were evaluated. The results allow concluding that the mixture fabricated with polymer modified binder presents a better behaviour in the mechanical properties evaluated. Also, the compaction energy and test temperature have greater influence on the mechanical properties in mixtures fabricated with penetration binder than those done with polymer modified binder.
\end{abstract}

Keywords: asphalt mixture, resilient modulus, rutting, mechanical properties 


\section{INTRODUCCIÓN}

Los pavimentos asfálticos constituyen un elemento básico de las infraestructuras viales de cada país y en éstos se realiza una inversión importante para su construcción y mantenimiento (De Solminihac, 1998). Sin embargo, estos pavimentos son solicitados durante su puesta en servicio, deteriorándose en el tiempo, por lo que es necesario para rentabilizar esta inversión, lograr que estos se comporten adecuadamente con la finalidad de que su ciclo de vida sea el previsto en su diseño. Para ello, se requiere caracterizar las propiedades de las mezclas asfálticas, con el propósito de realizar la elección más adecuada del tipo de ligante que permita que el pavimento presente un mejor comportamiento a lo largo de su vida útil.

Adicionalmente, las características reológicas de los ligantes como de las mezclas asfálticas hacen que su comportamiento sea completamente diferente frente a las solicitaciones producidas por el medioambiente y el tráfico, las cuales se manifiestan mediante variaciones térmicas y diferentes tipologías y velocidades de aplicación de las cargas, estableciendo para una mezcla asfáltica diferentes propiedades mecánicas según sean las condiciones de servicio (Pérez et al., 2006).

Conforme a lo anterior, la selección del tipo de ligante a utilizar en la mezcla asfáltica es de gran importancia, debido principalmente a la capacidad del ligante de aglomerar los materiales granulares junto con el comportamiento que pueda exhibir en el pavimento para las condiciones ambientales y tráfico en la que sea expuesto.

Dentro de las propiedades del ligante que entrega resistencia y tenacidad a la mezcla se encuentra la cohesión. Esta propiedad ha sido estudiada por diversos autores mediante el método de Caracterización Universal de Ligantes (UCL) desarrollado por la Universidad Politécnica de Cataluña. Estos autores concuerdan que la metodología UCL utilizada es idónea, puesto que analiza la cohesión del ligante como parte integrante de la mezcla asfáltica, a diferencia de las metodologías tradicionales mediante las cuales se analiza la cohesión solo desde el estudio del ligante en sí (Pérez et al., 1999; Bianchetto et al., 2002).

Otra propiedad que caracteriza a las mezclas asfálticas es su módulo de rigidez, el cual puede ser determinado en laboratorio mediante el ensayo de módulo resiliente a diferentes frecuencias y temperaturas. A su vez, el módulo de rigidez es un parámetro de diseño en los actuales modelos mecanicistas, el cual junto con el coeficiente de Poisson y el espesor de cada capa de la estructura del pavimento son los factores de entrada para determinar las tensiones y deformaciones horizontales y verticales producidas en el pavimento que permiten modelar su estructura y determinar su vida útil en función de la cantidad de aplicaciones de carga resistidas antes que el pavimento se fatigue (Asphalt Institute, 2007). Varios investigadores han estudiado esta propiedad de las mezclas asfálticas, por ejemplo Monismith et al. (1994), analizaron el efecto del nivel de esfuerzos y la variación de frecuencias de las cargas sobre las mezclas asfálticas, concluyendo que existe muy poca influencia de ambas variables analizadas sobre el valor del módulo resiliente resultante. Por otra parte, Elliot et al. (1992), realizaron un estudio para investigar los efectos de la variación de la granulometría de los agregados sobre las propiedades de la mezcla, concluyendo que no existían variaciones significativas en el módulo resiliente de la mezcla. A su vez, Bagampadde et al. (2006) señalan que para diferentes tipos de mezclas evaluadas experimentalmente, a medida que el ligante utilizado tiene una mayor penetración se obtienen módulos resilientes más altos, tanto para condiciones de ensayo en húmedo como en seco.

Roque y Buttlar (1992), establecen que la resistencia a tracción indirecta (RTI) es un parámetro importante de las mezclas asfálticas, puesto que caracteriza el fallo producido por tensiones de tracción en la fibra inferior de la capa asfáltica cuando ésta es solicitada por cargas de tráfico.

La resistencia a las deformaciones plásticas es una propiedad que debe ser analizada en el empleo de mezclas asfálticas densas, puesto que este tipo de mezclas son muy susceptibles a pequeñas variaciones en la composición de la mezcla o tipo de ligante empleado. Este parámetro fue estudiado por Ruiz y Pérez (2002) en mezclas convencionales, encontrando cambios significativos en la deformación permanente de acuerdo al tipo de ligante asfáltico utilizado, determinando a su 
vez, correlaciones entre la penetración, punto de ablandamiento con la velocidad de deformación de una mezcla asfáltica.

De acuerdo a lo expuesto, este estudio tiene por objetivo principal evaluar la influencia del tipo de ligante empleado en las propiedades mecánicas de una mezcla asfáltica densa. La mezcla seleccionada corresponde al tipo V-10 (Figura 1), acorde a las especificaciones chilenas (Ministerio de Obras Públicas, 2003a). La mezcla elegida es utilizada como capa de rodadura en una gran cantidad de países de Latinoamérica, y su elección para las pruebas experimentales radica principalmente en los fallos de ahuellamientos y fisuración presentados en este tipo de mezclas en los pavimentos flexibles. En las pruebas experimentales se evaluaron las propiedades de cohesión, módulo resiliente, tracción indirecta y deformaciones plásticas, para lo cual se utilizaron dos ligantes asfálticos convencionales de penetración (CA 60/80 y CA 80/10) y un ligante modificado con polímeros SBS (CA Modificado).

\section{METODOLOGÍA}

La metodología utilizada en este estudio se subdividió en tres fases. La primera fase consistió en realizar una adecuada caracterización de los materiales granulares y ligantes asfálticos a utilizar en la fabricación de la mezcla, para luego establecer el porcentaje de ligante óptimo de diseño de la mezcla de acuerdo al método Marshall. En la segunda fase se realizaron las pruebas de laboratorio conforme a las propiedades y variables que se deseaban evaluar. En la tercera fase se realizó el análisis de los resultados de las propiedades mecánicas evaluadas.

\section{Caracterización de los materiales y diseño de la mezcla}

Para la caracterización de los materiales utilizados en este estudio y el diseño de la mezcla asfáltica se utilizaron los métodos señalados en la normativa chilena (Ministerio de Obras Públicas, 2003b). Los resultados de los ensayos de caracterización del material granular triturado de origen fluvial y las características de los ligantes asfálticos utilizados en este estudio se presentan en las Tablas 1 y 2.

En la Figura 1 se ilustra la granulometría de la mezcla tipo $\mathrm{V}$-10 correspondiente a la utilizada en las pruebas experimentales.

En el diseño de la mezcla asfáltica se utilizó el procedimiento Marshall, establecido por el método 8.302.47 (Ministerio de Obras Públicas, 2003b), el cual se basa en el cálculo la cantidad de ligante óptimo para cada tipo de mezclas. Para esta investigación el contenido de ligante varío entre 5 y $7 \%$ para cada energía de compactación evaluada (50 y 75 golpes), resultando los porcentajes óptimos para cada tipo de ligante y parámetros de diseño de la mezcla, los que se ilustran en la Tabla 3.

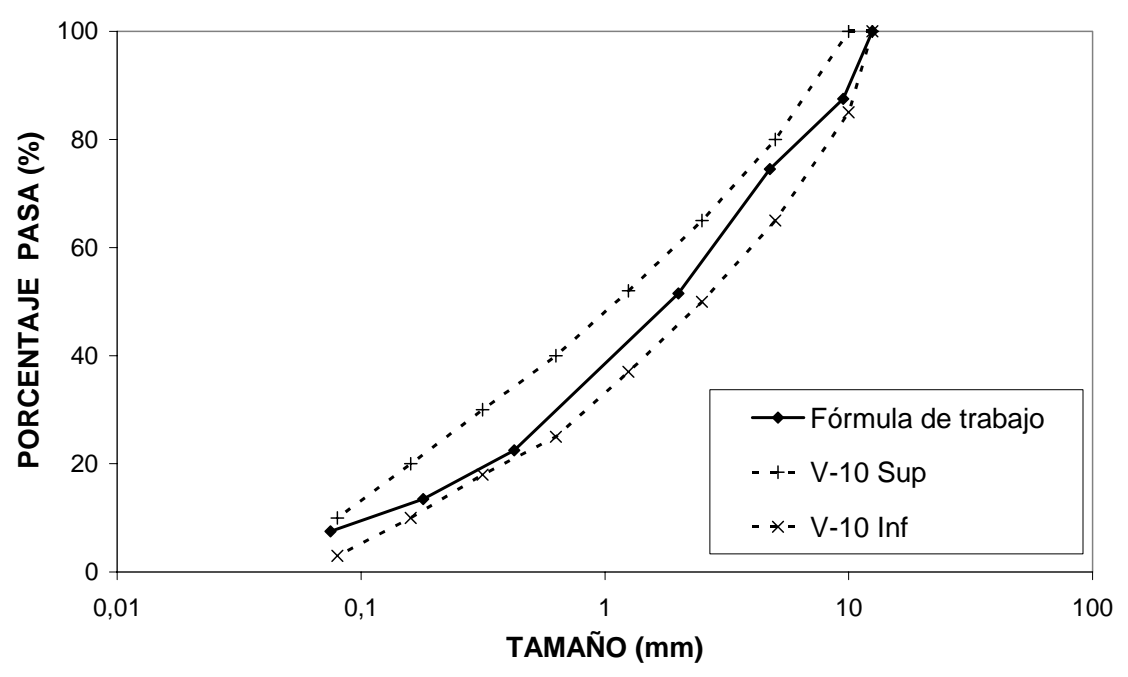

Fig. 1: Granulometría V-10 utilizada en el diseño de las mezclas evaluadas 
Tabla1: Ensayos de caracterización material granular

\begin{tabular}{l|c|c|c}
\hline Ensayo & Resultado & Especificación & Método \\
\hline Equivalente de arena & $72,93(\%)$ & Min. 50 (\%) & 8.202 .9 \\
Desgaste de Los Ángeles & $15,79(\%)$ & Máx. 25 (\%) & 8.202 .11 \\
Peso específico aparente granular grueso & $2,625\left(\mathrm{~g} / \mathrm{cm}^{3}\right)$ & - & 8.202 .20 \\
Absorción granular grueso & $0,47(\%)$ & - & 8.202 .20 \\
Peso específico aparente granular fino & $2,410\left(\mathrm{~g} / \mathrm{cm}^{3}\right)$ & - & 8.202 .21 \\
Absorción granular fino & $3,71(\%)$ & - & 8.202 .21 \\
\hline
\end{tabular}

Tabla 2: Ensayos de caracterización ligantes asfálticos utilizados

\begin{tabular}{l|c|c|c|l}
\hline \multirow{2}{*}{ Ensayo } & \multicolumn{3}{|c|}{ Tipo de ligante asfáltico } & \multirow{2}{*}{ Método } \\
\cline { 2 - 4 } & CA 60-80 & CA 80-100 & CA Modificado & \\
\hline Penetración $\left(25^{\circ} \mathrm{C}, 100 \mathrm{~g}, 0,1 \mathrm{~mm}\right)$ & 65 & 91 & 60 & 8.302 .3 \\
Punto de ablandamiento $\left({ }^{\circ} \mathrm{C}\right)$ & 52 & 49 & 68 & 8.302 .16 \\
Punto de inflamación $\left({ }^{\circ} \mathrm{C}\right)$ & 238 & 240 & 243 & 8.302 .9 \\
Punto de combustión $\left({ }^{\circ} \mathrm{C}\right)$ & 244 & 243 & 247 & 8.302 .9 \\
Perdida de masa RTFOT (\%) & 0,5 & 0,7 & 0,6 & 8.302 .33 \\
Recuperación elástica a 13 ${ }^{\circ} \mathrm{C}(\%)$ & - & - & 74 & 8.302 .19 \\
\hline
\end{tabular}

Tabla 3: Parámetros de diseño de mezclas, según procedimiento Marshall

\begin{tabular}{c|c|c|c|c|c|c|c|c}
\hline \multirow{2}{*}{$\begin{array}{c}\text { Tipo de } \\
\text { ligante }\end{array}$} & $\begin{array}{c}\text { Porcentaje } \\
\text { óptimo de } \\
\text { ligante } \\
\text { (\%) s/a }\end{array}$ & $\begin{array}{c}\text { Densidad } \\
\left(\mathrm{gr} / \mathrm{cm}^{3}\right)\end{array}$ & \multicolumn{2}{|c|}{$\begin{array}{c}\text { Estabilidad } \\
(\mathrm{N})\end{array}$} & \multicolumn{2}{|c|}{$\begin{array}{c}\text { Fluencia } \\
(0,25 \mathrm{~mm})\end{array}$} & \multicolumn{2}{|c}{$\begin{array}{c}\text { Huecos } \\
(\%)\end{array}$} \\
\cline { 2 - 9 } & Diseño & Diseño & Esp. & Diseño & Esp. & Diseño & Esp. \\
\hline CA 60-80 & 6,0 & 2,241 & 11.418 & & 12 & & 4,7 & \\
CA 80-100 & 6,25 & 2,228 & 9.525 & 9000 min. & 12 & $8-16$ & 5,0 & $4-6$ \\
CA Modif. & 6,0 & 2,208 & 9.601 & & 10 & & 4,5 & \\
\hline
\end{tabular}

\section{Pruebas de Laboratorio}

La evaluación de la cohesión de los ligantes asfálticos utilizados en el estudio se realizó por medio del procedimiento Universal de Caracterización de Ligantes, UCL, consistente en el cálculo de la masa conservada de probetas con una granulometría estándar, ilustrada en la Tabla 4, compactadas a 50 golpes por cara mediante el procedimiento Marshall y sometidas a abrasión en la Máquina de Desgaste de Los Ángeles por 300 ciclos. El contenido de ligante utilizado por el método UCL es de $4.5 \%$ sobre áridos. Se ensayaron series de tres probetas por cada ligante evaluado a las temperaturas de $0,10,20,30$ y $40^{\circ} \mathrm{C}$.

Tabla 4: Granulometría establecida por el método UCL

\begin{tabular}{c|c|c|c}
\hline Tamices UNE $(\mathrm{mm})$ & 5 & 2.5 & 0.63 \\
\hline \% Pasa & 100 & 20 & 0 \\
\hline
\end{tabular}

Para determinar el valor del módulo resiliente de las mezclas evaluadas se utilizó la normativa española NLT-360/91. El ensayo de módulo resiliente consiste en someter una probeta cilíndrica a pulsos de carga cíclica de compresión según un plano diametral y vertical de ella, midiéndose la deformación horizontal producida. El módulo resiliente total se calcula según la ecuación 1.

$$
E_{T}=\frac{P \cdot(v+0,27)}{h \cdot D_{t}}
$$

Donde, $\mathrm{E}_{\mathrm{T}}=$ módulo resiliente total, $\mathrm{MPa}$; $\mathrm{P}=$ amplitud pulso de carga, $\mathrm{N} ; \mathrm{v}=$ coeficiente de Poisson; $\mathrm{h}=$ espesor de la probeta, $\mathrm{mm}$; $\mathrm{D}_{\mathrm{T}}=$ deformación resiliente total, $\mathrm{mm}$. 
Las probetas ensayadas para la obtención del módulo resiliente fueron confeccionadas por el procedimiento Marshall para 2 energías de compactación, 50 y 75 golpes. Para cada tipo de ligante evaluado se ensayaron series de tres probetas a una frecuencia de $5 \mathrm{~Hz}$, y las temperaturas empleadas fueron 15,20 y $30^{\circ} \mathrm{C}$.

Para determinar el valor de la resistencia a tracción indirecta (RTI) de las mezclas evaluadas se utilizó la normativa española UNE-EN 12697-23. El ensayo consiste en la aplicación de una carga diametral a lo largo de la dirección del eje de una probeta circular, igual a la definida para el ensayo Marshall, a velocidad constante de desplazamiento de $50 \pm 2 \mathrm{~mm} / \mathrm{min}$ y a una temperatura de ensayo de $15^{\circ} \mathrm{C}$, hasta alcanzar a rotura de la probeta. Para la confección de las probetas se utilizaron dos energías de compactación, 50 y 75 golpes. Se ensayaron series de tres probetas por cada ligante evaluado.

La resistencia a la tracción indirecta se calcula mediante la ecuación 2:

$$
R T I=\frac{2 \cdot P}{\pi \cdot D \cdot h} \cdot 1000
$$

Donde, $\mathrm{RTI}=$ resistencia a la tracción indirecta, $\mathrm{kPa}$; $\mathrm{P}=$ carga máxima aplicada, $\mathrm{N} ; \mathrm{D}=$ diámetro de la probeta, $\mathrm{mm}$; $\mathrm{h}=$ altura de la probeta, $\mathrm{mm}$.

Para determinar la resistencia a las deformaciones plásticas de las mezclas evaluadas, se utilizó la normativa española NLT-173/00. El ensayo consiste en someter una probeta de mezcla asfáltica al paso de una rueda en condiciones determinadas de presión y temperatura, midiéndose periódicamente la profundidad de la deformación producida con la finalidad de determinar la velocidad de deformación de cada mezcla. Las muestras se fabricaron con la densidad determinada en probetas Marshall para 50 y 75 golpes, y se ensayaron a una presión vertical de $900 \mathrm{kPa}$, a una frecuencia de 42 ciclos por minuto y a una temperatura de $60^{\circ} \mathrm{C}$. Se ensayaron series de tres probetas por cada ligante y energía de compactación evaluada.

\section{RESULTADOS Y DISCUSIÓN}

Los resultados de los ensayos UCL a diferentes temperaturas para los tres tipos de ligante evaluados son presentados en la Figura 2. El asfalto modificado obtiene la mayor cohesión (mayor porcentaje de masa conservada) de los ligantes evaluados, a todas las temperaturas de ensayo, presentando muy poca influencia la variación de temperatura en la cohesión de este ligante. Para los asfaltos de penetración se observa una mayor influencia de la variación de la temperatura en la cohesión, presentando los mayores porcentajes de pérdidas a bajas temperaturas.

Los resultados ilustrados en la Figura 3 representan los módulos resilientes obtenidos para las mezclas fabricadas con los ligantes evaluados. Se observa una alta susceptibilidad al tipo de ligante, obteniendo módulos más altos la mezcla fabricada con el ligante asfáltico de menor penetración, CA 60-80, independientemente de la energía de compactación aplicada. A su vez, la mezcla fabricada con el ligante modificado presentó los menores módulos resilientes, siendo éstos del orden del $50 \%$ de los obtenidos por el CA 60-80. Concordando con lo expuesto por Mohammad y Paul (1992) para mezclas convencionales, los resultados señalan influencia directa de la temperatura en el módulo resiliente de las mezclas, apreciándose en la Figura 3, el descenso de este parámetro en función del aumento de la temperatura. Por otra parte, la energía de compactación influye en el módulo resiliente resultante para mezclas fabricadas con un mismo ligante, observándose un descenso del módulo a medida que decrece la energía para ligantes de penetración evaluados. Sin embargo, la diferencia en la energía de compactación aplicada en las mezclas fabricadas con el ligante modificado no muestra una influencia significativa como en las mezclas fabricadas con ligantes de penetración.

Los resultados que se observan en la Figura 4 indican una mayor RTI en la mezcla que se fabricó con el ligante modificado, y a su vez, esta mezcla registró el mayor desplazamiento para la 
resistencia máxima, ilustrando la mayor capacidad del ligante modificado para admitir deformaciones antes de su ruptura. Las RTI de las mezclas fabricadas con ligantes asfálticos de penetración obtuvieron valores similares, ligeramente superior para la mezcla fabricada con CA 60/80, diferenciándose en el desplazamiento registrado a resistencia máxima, presentando una mayor ductilidad la mezcla que se fabricó con CA 80/100. Los resultados expuestos para las mezclas confeccionadas con ligantes de penetración concuerdan levemente con lo determinado para mezclas densas por Kandhal y Chakraborty (1996), quienes determinaron para mezclas densas una correlación entre RTI y penetración del ligante, la cual establece una mayor RTI para ligantes con menor penetración.

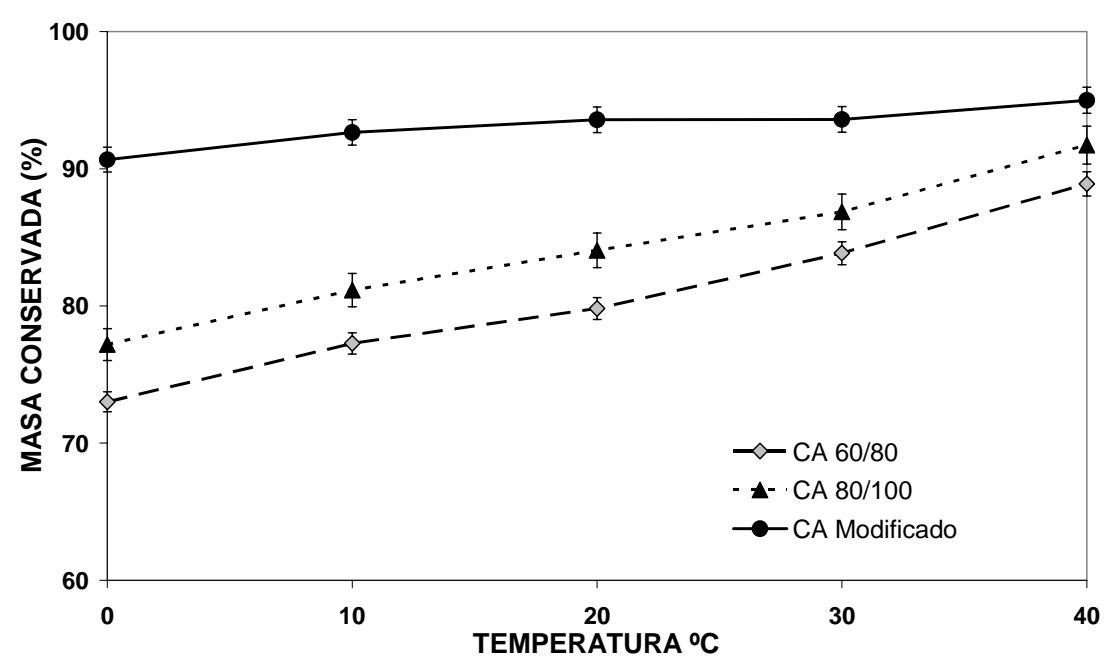

Fig. 2: Curvas de cohesión para los ligantes evaluados, ensayo UCL.

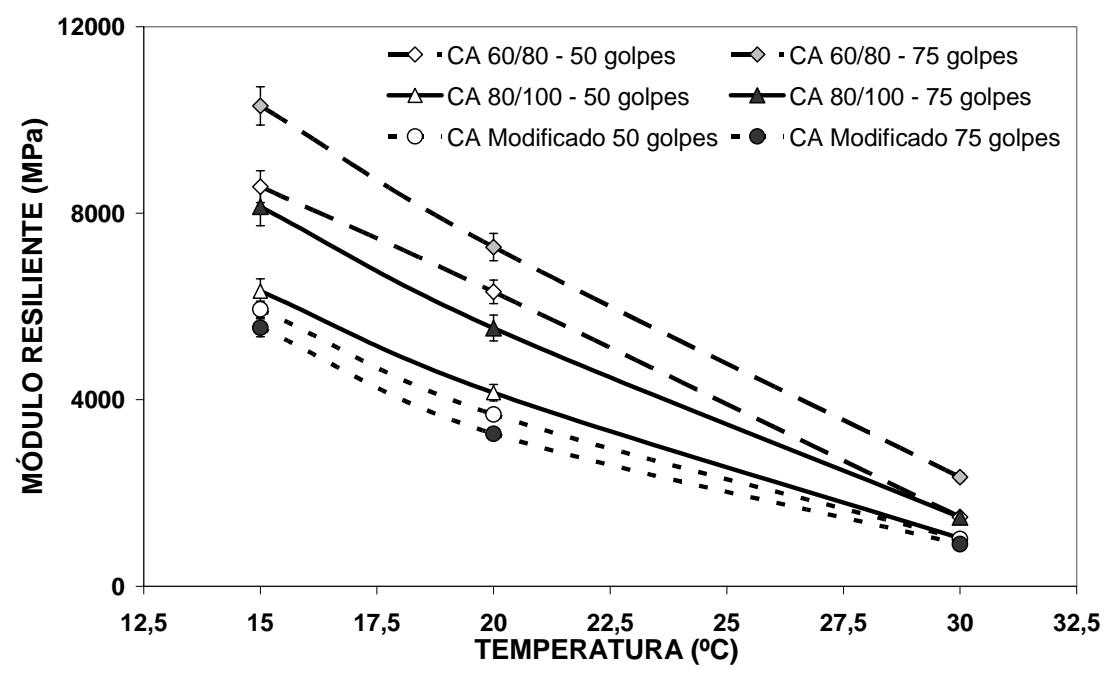

Fig. 3: Módulos resilientes de mezclas fabricadas con ligantes evaluados

Los resultados de resistencia a las deformaciones plásticas pueden ser observados en la Figura 5. Esta señala que la mezcla con menor deformación plástica la experimentó la fabricada con el ligante modificado, independiente de la energía de compactación, registrando una deformación similar para las distintas energías de compactación empleadas. Las mezclas fabricadas con ligantes de penetración presentaron una mayor influencia de la energía de compactación empleada, observándose una mayor deformación plástica en ambas energías de compactación, 50 y 75 golpes, en la mezcla que se fabricó con el ligante de mayor penetración, CA 80/100.

En la Figura 5, a su vez, se presentan las velocidades de deformación en el intervalo de 105 a 120 minutos para los ligantes evaluados en las mezclas. Se observa la menor velocidad de deformación 
en la mezcla fabricada con ligante modificado, concordando con lo investigado para mezclas convencionales por Ruiz y Pérez (2002). Por otra parte, la mayor velocidad de deformación la presenta el ligante de mayor penetración. Además, se pone de manifiesto que todos los ligantes evaluados en las mezclas cumplen con las velocidades de deformación máxima establecida por la normativa española, de $20 \mu \mathrm{m} / \mathrm{min}$ (Ministerio de Fomento, 2002).

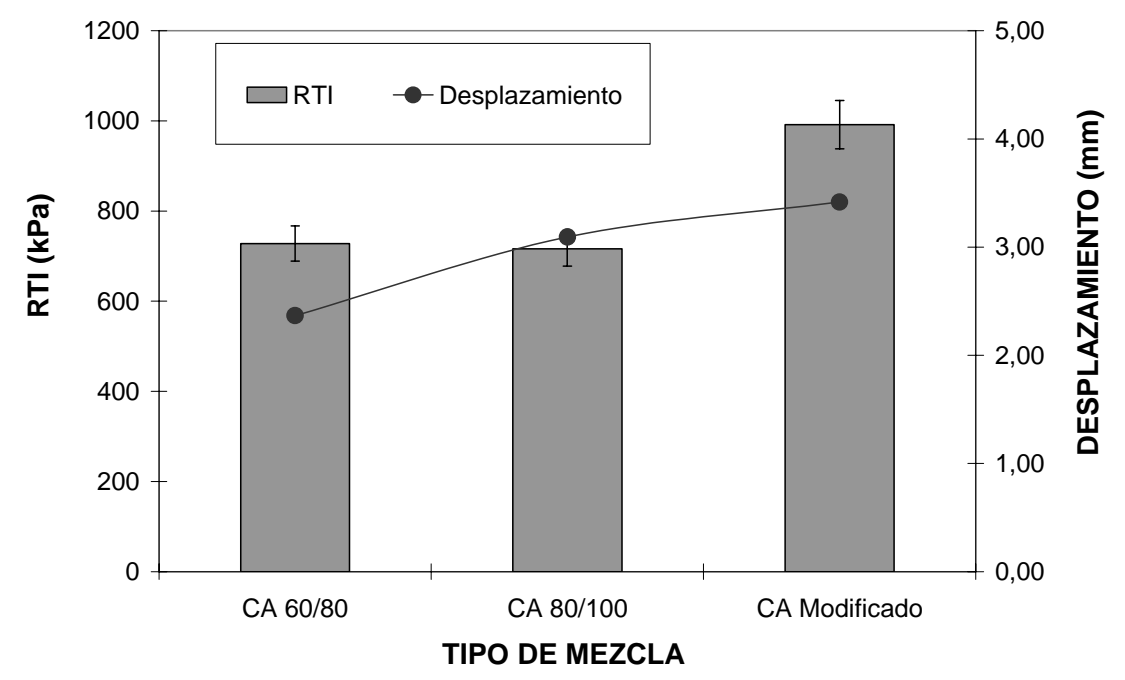

Fig. 4: Resistencia a tracción indirecta de mezclas fabricadas con ligantes evaluados
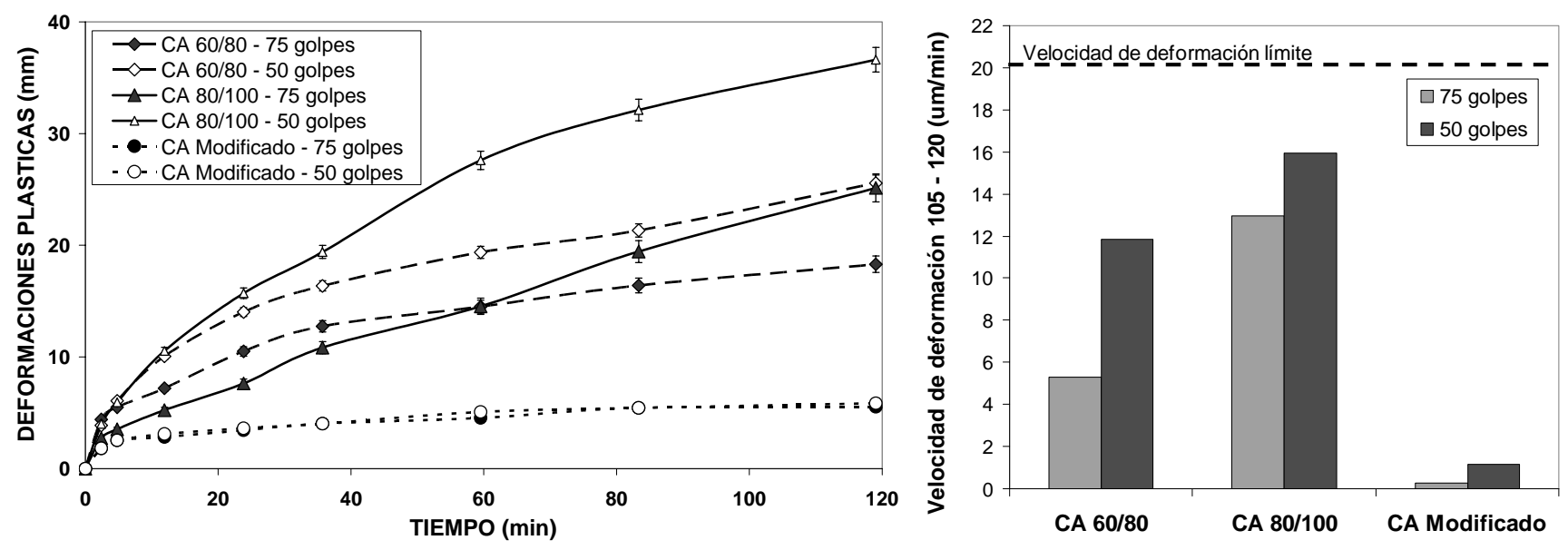

Fig. 5: Deformaciones plásticas de mezclas fabricadas con ligantes evaluados

\section{CONCLUSIONES}

Las pruebas de laboratorio realizadas señalan que el ligante modificado con polímeros SBS registra un mejor comportamiento frente a las propiedades mecánicas de cohesión, resistencia a tracción indirecta y deformaciones plásticas, a las temperaturas y energías de compactación evaluadas en este estudio. Por otra parte, del análisis de los módulos resilientes se establece la susceptibilidad de este parámetro ante el ligante empleado. A su vez la energía de compactación y la temperatura de ensayo muestran una influencia mayor en mezclas fabricadas con ligantes de penetración respecto de las fabricadas con el ligante modificado para las propiedades mecánicas evaluadas.

De acuerdo a lo anterior, el ligante modificado con polímeros SBS es el que ofrece mejores prestaciones para la mezcla evaluada, estableciendo la importancia de la elección del tipo de ligante para obtener un mejor comportamiento estructural y funcional cuando un pavimento es solicitado por distintas condiciones de tráfico y medioambientales durante su puesta en servicio. 


\section{REFERENCIAS}

Asphalt Institute, The Asphalt Handbook MS-4, $7^{\text {th }}$ edition, Asphalt Institute, 281-290, USA (2007).

Bagampadde, U., Isacsson, U. y B.M. Kiggundu; Impact of bitumen and aggregate composition on stripping in bituminous mixtures, Journal Materials and Structures: 287, 303-315 (2006)

Bianchetto, H. y otros cuatro autores; Nuevas investigaciones sobre ligantes bituminosos en Argentina a partir del método de caracterización UCL, Carreteras: 124, 36-48 (2002).

De Solminihac, H.; Gestión de Infraestructura Vial, $2^{\text {a }}$ edición, Ediciones Universidad Católica de Chile, 3-18, Santiago, Chile (1998).

Elliot, R.P., M.C. Ford, y M. Ghanim; Effect of Aggregate Gradation Variation on Asphalt Concrete Mix Properties, Transportation Research Record: 1317, 52-60 (1992).

Kandhal, P.S. y S. Chakraborty; Effect of Asphalt Film Thickness on Short - and Long - Term Aging of Asphalt Paving Mixtures, Transportation Research Record: 1535, 83-90 (1996).

Mohammad, L.N. y H.R. Paul; Evaluation of a New Indirect Tension Test Apparatus, Transportation Research Record: 1353, 62-68 (1992).

Monismith, C.L., B. Tsai y A.A. Tayebali; Stiffness of Asphalt Aggregate Mixes, Report prepared for SHRP Project A-338, Asphalt Research Program, Institute of Transportation Studies, University of California, Berkeley, 1-99, Washington DC, USA (1994).

Pérez, F.E., R. Miró, H.A. Khalid, y C.M. Walsh; Procedimientos funcionales para la caracterización de ligantes. Métodos SHRP y UCL, Carreteras: 100, 39-48 (1999).

Pérez, F.E., R. Miró, y A. Martínez; Estudio, Diseño y Control de Mezclas Bituminosas, $1^{\mathrm{a}}$ edición, Asociación Española de Carreteras, 11-34, Madrid, España (2006).

Roque, R. y W.G., Buttlar; The Development of Measurement and Analysis System to Accurately Determine Asphalt Concrete Properties Using The Indirect Tensile Mode, Asphalt Paving Technology: 63, 305-332 (1992).

Ruiz, A. e I. Pérez; Influencia de los Betunes en las Deformaciones Plásticas de las Mezclas Bituminosas, Cedex Centro de Estudios y Experimentación de Obras Públicas, 79-98, Madrid, España (2002).

Ministerio de Obras Públicas, Manual de Carreteras, 5, 251-272, Dirección de Vialidad - MOP Chile, Santiago, Chile (2003a).

Ministerio de Obras Públicas, Manual de Carreteras, 8, 327-725, Dirección de Vialidad - MOP Chile, Santiago, Chile (2003b)

Ministerio de Fomento, PG-3: Pliego de Prescripciones Técnicas Generales para Obras de Carreteras y Puentes, $3^{a}$ edición, Ediciones Liteam, 339-408, Madrid, España (2002). 